\title{
Identification of genes functionally involved in the detrimental effects of mutant histone H3.3-K27M in Drosophila melanogaster
}

\author{
Johannes Berlandi," Amel Chaouch," Nicolas De Jay, Isabel Tegeder, Katharina Thiel, \\ Margret Shirinian, Claudia L. Kleinman, Astrid Jeibmann, Paul Lasko, Nada Jabado," and \\ Martin Hasselblatt ${ }^{\#}$
}

\begin{abstract}
Institute of Neuropathology, University Hospital Münster, Münster, Germany (J.B., I.T., K.T., A.J., M.H.); Department of Biology (A.C., P.L.) and Department of Human Genetics, McGill University, Montreal, Quebec, Canada (N.D.J., C.L.K.); The Lady Davis Institute, Jewish General Hospital, Montreal, Quebec, Canada (N.D.J., C.L.K.); Department of Experimental Pathology, Immunology, and Microbiology Faculty of Medicine, American University of Beirut, Beirut, Lebanon (M.S.); Department of Paediatrics, McGill University and the McGill University Health Center Research Institute, Montreal, Quebec, Canada (N.J.)
\end{abstract}

Corresponding Author: Martin Hasselblatt, M.D., Institute of Neuropathology, University Hospital Münster, Pottkamp 2, 48149 Münster, Germany (hasselblatt@uni-muenster.de).

"Authors contributed equally.

\begin{abstract}
Background. Recurrent specific mutations in evolutionarily conserved histone 3 (H3) variants drive pediatric highgrade gliomas (HGGs), but little is known about their downstream effects. The aim of this study was to identify genes involved in the detrimental effects of mutant H3.3-K27M, the main genetic driver in lethal midline HGG, in a transgenic Drosophila model.

Methods. Mutant and wild-type histone H3.3-expressing flies were generated using a $\varphi$ C31-based integration system. Genetic modifier screens were performed by crossing H3.3-K27M expressing driver strains and 194 fly lines expressing short hairpin RNA targeting genes selected based on their potential role in the detrimental effects of mutant H3. Expression of the human orthologues of genes with functional relevance in the fly model was validated in H3-K27M mutant HGG.

Results. Ubiquitous and midline glia-specific expression of H3.3-K27M but not wild-type H3.3 caused pupal lethality, morphological alterations, and decreased H3K27me3. Knockdown of 17 candidate genes shifted the lethal phenotype to later stages of development. These included histone modifying and chromatin remodeling genes as well as genes regulating cell differentiation and proliferation. Notably, several of these genes were overexpressed in mutant H3-K27M mutated HGG.

Conclusions. Rapid screening, identification, and validation of relevant targets in "oncohistone" mediated pathogenesis have proven a challenge and a barrier to providing novel therapies. Our results provide further evidence on the role of chromatin modifiers in the genesis of H3.3-K27M. Notably, they validate Drosophila as a model system for rapid identification of relevant genes functionally involved in the detrimental effects of H3.3-K27M mutagenesis.
\end{abstract}

\section{Key Points}

1. To identify pathways involved in the biology of histone $3(\mathrm{H} 3)$ in pediatric high-grade gliomas, a fly model was developed.

2. Here we show that a set of genes involved in chromatin remodeling and development is functionally implicated in the effects of H3.3-K27M. 


\section{Importance of the Study}

Pediatric HGGs are malignant brain tumors with devastating outcomes. These tumors are driven by specific mutations in $\mathrm{H} 3$ variants, but little is known about downstream genes that are mis-regulated upon expression of mutant $\mathrm{H} 3$, and how changes in gene expression contribute to tumorigenesis. Taking advantage of the high evolutionary conservation of $\mathrm{H} 3$ variants, we developed a fly model to identify genes and signaling pathways involved in the detrimental effects of mutant
H3. Here we show both that ubiquitous and glial-specific expression of oncohistones produces deleterious phenotypes, and that a small, highly specific, set of genes involved in chromatin remodeling and development is functionally implicated in the effects of H3.3$\mathrm{K} 27 \mathrm{M}$. This approach could be extended to investigate other oncogenic mutant histones and identify relevant therapeutic targets in $\mathrm{H} 3 \mathrm{~K} 27 \mathrm{M}$ pathogenesis.
High-grade gliomas (HGGs) are the most common type of primary brain cancer in adults. In children, they account for $15 \%$ of brain cancers and include diffuse intrinsic pontine gliomas, which are particularly lethal neoplasms with a median survival of only 10 months postdiagnosis. Despite histological similarities, and an equally devastating and fatal outcome, pediatric and adult HGGs are molecularly distinct diseases. While increasing evidence suggests that adult malignant gliomas are genetically and epigenetically heterogeneous, we and others identified recurrent somatic gain-of-function mutations in 2 specific residues of histone 3 (H3) variants to be commonly altered in pediatric and young adult HGGs, where they act as major drivers in oncogenesis. ${ }^{1-3}$ We identified 2 mutually exclusive recurrent mutations in $\mathrm{H} 3$ variants, p.Lys27Met (K27M) in $\mathrm{H} 3.3$ and H3.1/2, and p.Gly34Arg/Val (G34R/V) in H3.3. Interestingly, these mutations are also mutually exclusive with the recurrent mutations in isocitrate dehydrogenase (IDH) identified in many young adult gliomas. Similar to IDH mutants, these "oncohistones" require association with additional specific genetic alterations for full progression to gliomas to occur. ${ }^{2,4-8}$ We further showed that different $\mathrm{H} 3$-associated mutations occur in different patient age groups and give rise to HGGs in specific anatomic compartments in the brain. Indeed, $\mathrm{K} 27 \mathrm{M}$ arises in $~ 80 \%$ of midline $\mathrm{HGGs}$, and recently diffuse midline glioma H3-K27M mutant (World Health Organization [WHO] grade IV) has been recognized as a distinct tumor entity. ${ }^{9}$ In contrast, mutations affecting the H3K36 mark (SETD2, H3.3G34R/V) ${ }^{10-12}$ occur in $42 \%$ of cerebral hemisphere HGGs, indicative that oncohistones arise during specific windows of development.

Histones package and organize DNA at the fundamental chromatin unit, the nucleosome. ${ }^{13}$ The nucleosome is composed of a hetero-octamer of histones comprising a tetramer $(\mathrm{H} 3-\mathrm{H} 4)_{2}$ flanked by $2 \mathrm{H} 2 \mathrm{~A}-\mathrm{H} 2 \mathrm{~B}$ dimers, around which 147 bp of DNA is wrapped. ${ }^{13}$ The dynamics of this organization permit the compaction of the genome, while enabling all cellular processes operating on DNA to occur. DNA condensation is regulated by posttranslational modifications of protruding N-terminal histone tails (eg, methylation, acetylation, phosphorylation, ubiquitinylation), which enable dynamic and cell type-specific regulation of genes. ${ }^{14}$ H3.3-K27M affects the SET domain of enhancer of zeste homolog 2, leading to a drastic decrease in the levels of H3K27 tri- and dimethylation in cells, while H3.3-G34R/V may impair the methylation of the downstream H3.3-K36 mark. ${ }^{15}$ These effects on histone methylation potentially cause transcriptional dysregulation and alter the pattern of DNA methylation, ${ }^{2,16-18}$ but little is known about the specific downstream events they produce and the function of the affected genes and signaling pathways in driving tumorigenesis.

This current lack of understanding of the effects of histone mutagenesis impedes the design of effective therapies for HGGs. ${ }^{2,16-20}$ Another major challenge to developing such therapies is the paucity of preclinical models that recapitulate the pathogenicity of these oncohistones. ${ }^{21}$ Importantly, histones are among the most evolutionarily conserved proteins, and the $\mathrm{H} 3$ variants H3.1 and H3.3 are identical in humans and the fruit fly Drosophila melanogaster. ${ }^{13}$ Drosophila has proven to be a powerful genetic model to study human diseases, including brain tumors, ${ }^{22}$ and to decipher specific genes and pathways whose regulation is affected by specific perturbations, including histone mutagenesis. ${ }^{23}$ We thus aimed to identify genes functionally involved in the detrimental effects of H3.3-K27M mutants in vivo. To this end, a Drosophila model expressing human mutated K27Mor G34R-H3.3 or expressing wild-type histone H3.3 was established. Here we show both that ubiquitous and cell type-specific expression of oncohistones produces deleterious phenotypes and that genes involved in chromatin remodeling and development are functionally implicated in the detrimental effects of H3.3-K27M.

\section{Materials and Methods}

\section{Fly Stocks and Genetics}

Mutant and wild-type histone H3.3-expressing flies (UASH3.3 wild-type, UAS-H3.3-K27M, UAS-G34R-H3.3) were generated using a $\varphi \mathrm{C} 31$-based integration system. All strains were kept at $25^{\circ} \mathrm{C}$ and $60 \%$ humidity. Flies were crossed against cell type-specific GAL4 driver lines (obtained from the Bloomington Drosophila Stock Center and the Vienna Drosophila Resource Center) to activate expression of UAS constructs.

- Act5C-GAL4/CyO::Deformed GFP (kindly provided by Christian Klämbt)

- slit-GAL4/CyO,Tb (kindly provided by Christian Klämbt) 
- elav-GAL4 (BDSC: 458)

- repo-GAL4 (kindly provided by Christian Klämbt)

\section{Immunofluorescence}

Third instar larvae were fixed in $3.7 \%$ formalin overnight at $4^{\circ} \mathrm{C}$. Subsequently, larval brains were dissected and stained using various primary antibodies (mouse anti-Slit [1:25, C555.6D, Developmental Studies Hybridoma Bank], rabbit anti-Tri-Methyl-Histone H3 [Lys27, 1:500, C36B11, Cell Signaling], and rabbit anti-Wrapper [1:25, 10D3, Developmental Studies Hybridoma Bank]). Secondary antibodies (goat $\mathrm{Cy}^{\mathrm{TM}} 3$ anti-mouse [Dianova], goat Alexa Fluor 488 anti-rabbit [Thermo Fisher Scientific], and goat Alexa Fluor 647 anti-rabbit [Thermo Fisher Scientific]) were used at dilution of 1:1000. Brains were mounted in RotiMount FluorCare (Carl Roth) and analyzed using a Zeiss LSM 710 microscope.

\section{Confocal Microscopy and Image Analysis}

For image acquisition of larval brains, after whole mount fluorescent staining, an Axio Imager M2 with an LSM 700 confocal unit (Zeiss) was used (lenses: 10x Plan Apo, numerical aperture $[\mathrm{NA}]=0.45 ; 20 \mathrm{x}$ Plan Apo, $\mathrm{NA}=0.8$; 40x Plan Apo, NA = 1.4). Zen 2009 software (Zeiss) and the ImageJ software (available at http://imagej.nih.gov/ij/) were used for image processing. For quantification of the signal intensity of immunofluorescence images, the mean gray value throughout the larval brain was measured using ImageJ software.

\section{Western Blot}

Proteins were extracted from third instar larval brains. Approximately 100 larval brains were frozen for $5 \mathrm{~min}$ at $-80^{\circ} \mathrm{C}$ in a $1.5 \mathrm{~mL}$ reaction tube. Subsequently, $100 \mu \mathrm{L}$ cold radioimmunoprecipitation assay buffer $(0.1 \%$ sodium dodecyl sulfate [SDS]; $1 \%$ NP-40; $0.5 \%$ sodium deoxycholate; 50 mM Tris, $\mathrm{pH}$ 8; $150 \mathrm{mM} \mathrm{NaCl}$; 1:100 phenylmethylsulfonyl fluoride; 1:1000 proteinase-inhibitor) was added and larval brains were ground with pestles. Samples were centrifuged for $5 \mathrm{~min}$ at $13000 \mathrm{rpm}$ and $4^{\circ} \mathrm{C}$. The supernatant was transferred into a fresh reaction tube and samples were stored at $-20^{\circ} \mathrm{C}$ until further usage. Protein extracts were heated for $5 \mathrm{~min}$ at $95^{\circ} \mathrm{C}$ in sample buffer and run on a $10 \%$ SDS-polyacrylamide gel electrophoresis gel. Separated proteins were blotted using nitrocellulose and incubated with mouse anti-Slit antibody (C555.6D, 1:200, Developmental Studies Hybridoma Bank) and goat Alexa Fluor 488 anti-mouse (1:3000, Thermo Fisher Scientific). Stained protein bands were visualized using a ChemiDoc MP imaging system (Bio-Rad).

\section{Fly Genetic Modifier Screen}

Cell type-specific screening strains were generated by balancing second and third chromosomes carrying the GAL4 and UAS construct, respectively. Ubiquitous or midline glial expression of transgenic H3.3-K27M was achieved using actin-GAL4 or slit-GAL4 driver strains, respectively.

$$
\begin{aligned}
& +\frac{\text { actin }-G A L 4}{C y O, T b} ; \frac{U A S-K 27 M-H 3.3}{\text { tubulin }- \text { Gal80 }} \\
& +\frac{\text { slit }-G A L 4}{C y O, T b} ; \frac{U A S-K 27 M-H 3.3}{\text { tubulin }- \text { Gal80 }}
\end{aligned}
$$

Genetic modifier screens were conducted by crossing screening strains to RNA interference (RNAi) lines (GD or KK stocks obtained from Vienna Drosophila Resource Center and the Bloomington Drosophila Stock Center) to test for a modification of detrimental effects of H3.3K27M expression. RNAi lines of 194 candidate genes (Supplementary Table 1) were investigated regarding a negative or positive shift of lethality induced by H3.3-K27M expression. To control for GAL4 dosage effects, all screening strains were crossed to UAS-mCherry-RNAi. Positive shifts were verified in triplicates. To guarantee a sufficient amount of animals affected by short hairpin (sh)RNA knockdown, only crosses resulting in at least 50 control animals were scored.

\section{RNA Sequencing of Human Tumor Samples}

For gene expression analyses in human HGGs, we used a recently published RNA-seq dataset consisting of 5 H3-K27M HGGs, 4 H3 wild-type HGGs, and 3 normal brain samples. ${ }^{21}$ Raw sequencing reads were trimmed using Trimmomatic ( $\mathrm{v} 0.32)^{24}$ to remove the first 4 bases of each read, low-quality bases at the start and end of reads (phred33<30), as well as Illumina adaptor sequences using palindrome mode. A sliding window of 4 nucleotides was used to cut reads when the average quality of the window fell too low (phred33 $<30$ ). Reads shorter than 30 nucleotides after trimming were discarded. The high-quality reads resulting from this procedure were aligned to the human reference genome (GRCh37) using STAR (v2.30e) and default settings. Multimapping reads (ie, mapping to more than 10 genomic locations) were discarded.

Gene expression was quantified with featureCounts (v1.4.4) using Ensembl's ensGene annotation set. Normalization (median of ratios) and identification of differentially expressed genes were performed using DESeq2 (v1.14.1) using the negative binomial distribution to determine statistical significance. To quantify the enrichment of the set of genes identified in Drosophila across human samples, we used single-sample Gene Set Enrichment Analysis (ssGSEA). ${ }^{25}$ Statistical significance of the difference in distribution of gene expression levels (in reads per kilobase per million [RPKM]) in H3-K27M HGG, H3 wildtype HGG, and normal brain samples was determined using Kruskal-Wallis ANOVA followed by Dunn's test for multiple comparisons.

\section{Immunohistochemistry of Human Tumor Samples}

For immunohistochemistry, tissue sections from formalinfixed paraffin-embedded samples of 8 histone H3(K27M) mutated HGGs as well as 9 histone $\mathrm{H} 3$ wild-type HGGs 
were stained using antibodies directed against cyclin D1 (CCND1) (1:20, RM-9104, Thermo Fisher Scientific), B lymphoma Moloney-murine leukemia virus insertion region 1 homolog (BMI1) (1:500, EPR3745[2], Abcam), and high mobility group box 2 (HMGB2) (1:200, PA5-62773, Thermo Fisher Scientific) on an automated staining system (Dako). Tumor samples were collected from the archives of the Institute of Neuropathology Münster. Our tumor bank received local ethical committee approval (ethics committee of the University Hospital Münster), and patients had given informed consent for scientific use of the archival materials.

\section{Results}

\section{Expression of Mutant Histone H3.3 Causes} Lethality in Drosophila Melanogaster

Transgenic expression of mutant histone $\mathrm{H} 3.3$ in Drosophila melanogaster resulted in different phenotypes for H3.3 mutants, while control expression of human wildtype H3.3 had no effect on the tested Drosophila strains (Table 1). Ubiquitous expression of H3.3-K27M or H3.3G34R caused lethality at the pupal stage. Expression of H3.3-K27M in glial cells (repo-GAL4) and midline glia (slit$G A L 4$ ) resulted in a lethal phenotype at the pupal stage and the late pupal stage, respectively, in contrast to expression of H3.3-G34R, which did not produce a phenotype using these drivers. No phenotype was observed using neuronal GAL4 driver strains for either mutant H3.3 (Table 1).

\section{Expression of Mutant H3.3-K27M in Drosophila Melanogaster Causes Morphological Changes and Reproduces Epigenetic Alterations Seen in Human Tumors}

Ubiquitous expression of H3.3-K27M but not wild-type or H3.3-G34R resulted in the formation of melanotic masses in the larval hemocoel (Fig. 1A-D, Supplementary Fig. $1 \mathrm{~A}, \mathrm{~B})$. Under these conditions, as expected, H3.3-K27M caused a global reduction of histone $\mathrm{H} 3 \mathrm{~K} 27$ trimethylation (H3K27me3; Fig. 1E-H, Supplementary Fig. 1C). Cell typespecific expression of $\mathrm{H} 3.3-\mathrm{K} 27 \mathrm{M}$ in midline glia (but not wild-type or H3.3-G34R) also strongly reduced H3K27me3 in the larval brain, H3K27me3 being only detectable in the periphery of the ventral nerve cord and essentially absent in midline glia (Fig. 2A-D). Expression of H3.3-K27M (but not wild-type or H3.3-G34R) also altered the distribution of Slit protein, an important midline glial-derived inhibitor of axonal migration (Fig. 2E-H). Distribution of midline glial marker Wrapper remained unchanged, suggesting perturbed localization of (extracellular) Slit protein rather than perturbed migration of midline glia cells (Supplementary Fig. 2). Furthermore, expression of Slit protein in the larval brain was found to be increased upon H3.3-K27M expression on western blot (Supplementary Fig. 3). These data are consistent with findings in human HGGs as K27M mutagenesis characterizes midline tumors of glial lineage where Slit, a neural axonal guidance factor previously involved in glioma invasion, ${ }^{26}$ is overexpressed in K27M HGGs. As the wing is commonly examined to study proliferation in Drosophila, we also expressed histone H3.3-K27M under the control of engrailed, which resulted in increased proliferation of the larval wing disc (Supplementary Fig. 4).

\section{Genes Involved in the Detrimental Effects of H3.3-K27M Expression}

We focused on further investigating the effects of H3.3$\mathrm{K} 27 \mathrm{M}$ expression in midline glia, or ubiquitously, because of the relevance to human disease of the phenotypes we obtained. To identify genes functionally involved in the detrimental effects of H3.3-K27M expression, we performed a modifier screen on the fly lines. To this effect we selected 194 candidate genes known to play a role in glial cell biology and/or to affect H3.3 function, including its posttranslational modifications and its incorporation in the chromatin (Supplementary Table 1). We expressed shRNA targeting each of these genes in the transgenic H3.3-K27M fly, and examined whether the lethal phenotype of H3.3$\mathrm{K} 27 \mathrm{M}$ flies was shifted to earlier or later stages of development. Under control conditions, ubiquitous H3.3-K27M expression in the screening strain (Act5C > UAS- H3.3K27M /UAS-mCherry-RNAi) caused lethality at the early pupal stage, with $21 \%$ (median range, $18-22 \%$ ) of animals failing to pupate and showing incomplete or deformed pupal shells (Fig. 3A, control RNAi). From this starting point, shRNA knockdown of 8 of the 194 candidate genes shifted pupal lethality to later stages of development (ie, absence of pupation defects; Fig. 3A, Table 2). These included 6 genes encoding histone modifying and chromatin remodeling proteins: high mobility group genes $\mathrm{HMG}$ protein $\mathrm{Z}(\mathrm{HmgZ})$ and HMG protein D $(\mathrm{HmgD})$, polycomb

Table 1 Effect of mutant H3.3 expression in Drosophila melanogaster*

$\begin{array}{llll}\text { GAL4 Expression } & \text { H3.3-K27M } & \text { G34R-H3.3 } & \text { H3.3Wild-Type } \\ \text { Ubiquitous (Act5C-GAL4) } & \text { Pupal lethal } & \text { Pupal lethal } & \text { Viable } \\ \text { Glial cells (repo-GAL4) } & \text { Pupal lethal } & \text { Viable } & \text { Viable } \\ \text { Midline glial cells (slit-GAL4) } & \text { Pupal lethal, eclosure defects } & \text { Viable } & \text { Viable } \\ \text { Neurons (elav-GAL4) } & \text { Viable } & \text { Viable } & \text { Viable }\end{array}$

*Effect of ubiquitous and cell type-specific transgenetic expression of mutant H3.3 (H3.3-K27M and G34R-H3.3) compared with H3.3 wild-type (control) in Drosophila melanogaster. 
Wild type

A

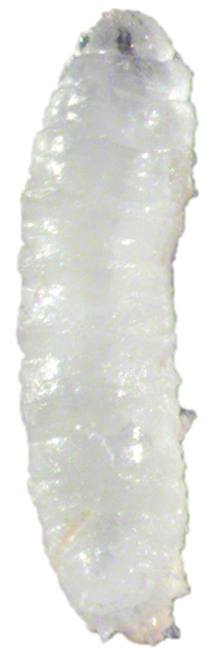

E
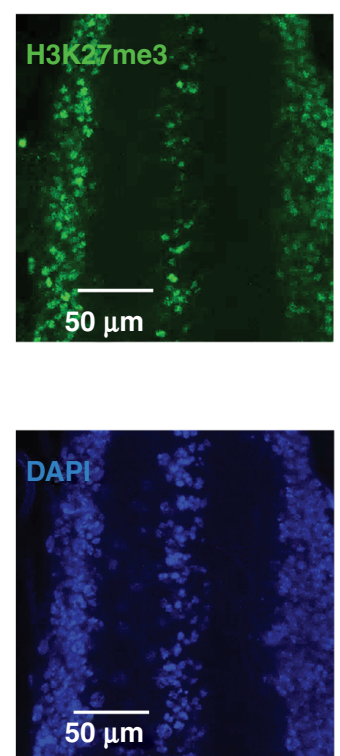

H3.3 WT

B

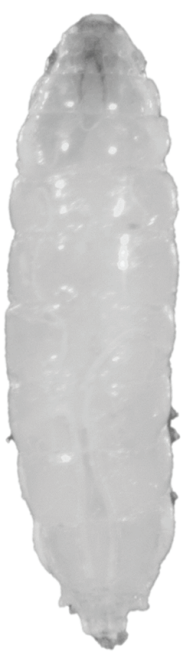

F
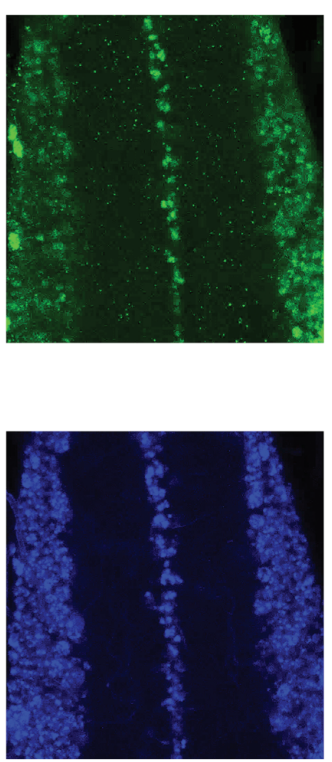

H3.3-K27M

C

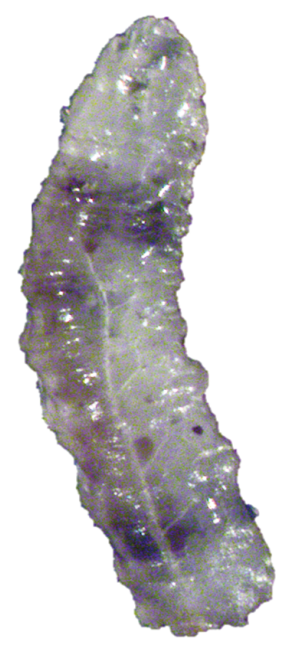

G
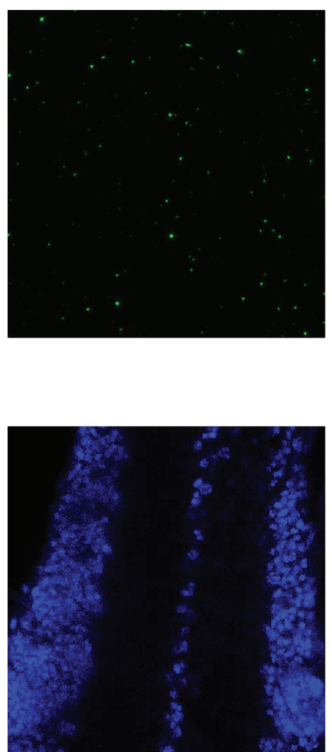

H3.3-G34R

D

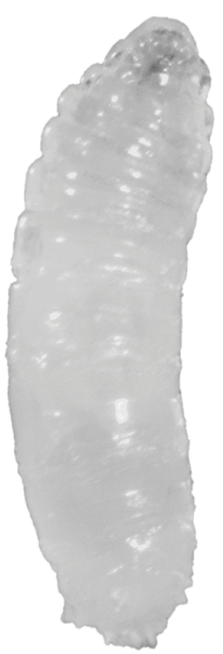

$\mathrm{H}$
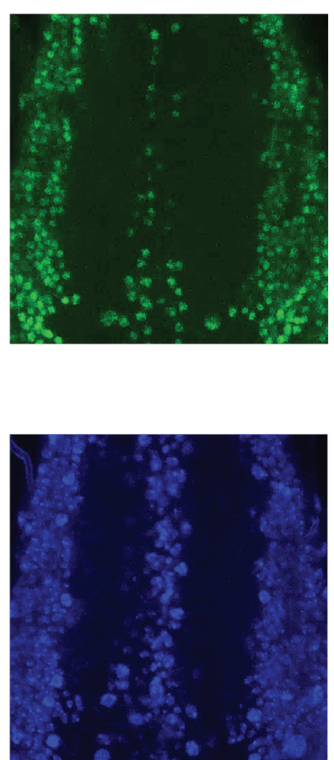

Fig. 1 Phenotype upon ubiquitous expression of wild-type and mutant H3.3 in Drosophila. Phenotype of third instar control larvae (A) compared with ubiquitous expression of H3.3 wild-type (WT, B), H3.3-K27M (C), and H3.3-G34R (D). Note formation of melanotic masses only upon ubiquitous H3.3-K27M expression. H3K27me3 expression in third instar larval wild-type brains (control, E) compared with ubiquitous expression of H3.3 wildtype (WT, F), H3.3-K27M (G), and H3.3-G34R (H). Note complete loss of H3K27me3 only upon ubiquitous H3.3-K27M expression. Nuclear staining by $4^{\prime}, 6^{\prime}$-diamidino-2-phenylindole serves as control. For each condition, at least 5 animals were examined.

repressive complex 1 (PRC1) component suppressor of zeste $2(S u(z) 2)$, testis-specifically expressed bromodomain containing protein 3 (tbrd-3), elongator acetyltransferase complex subunit 3 (Elp3), and CG8223, as well as downstream genes Ephrin and trol (terribly reduced optic lobes). In contrast, shRNA knockdown of 41 candidate genes worsened the Act5C > H3.3-K27M phenotype and produced complete larval lethality. These genes included PRC2 members $E(z)$ (enhancer of zeste), Caf1-55 (chromatin assembly factor 1, p55 subunit), $S u(z) 12$, and jing. In an 
Wild type

A
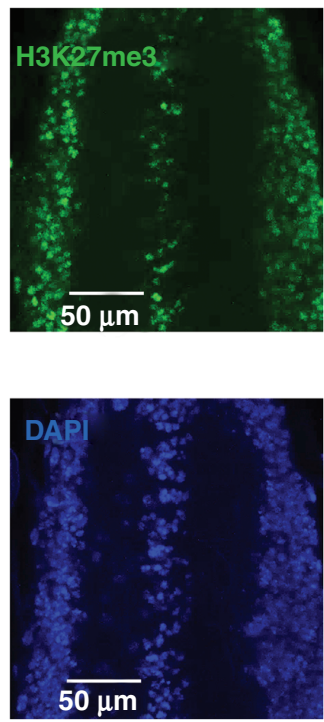

E
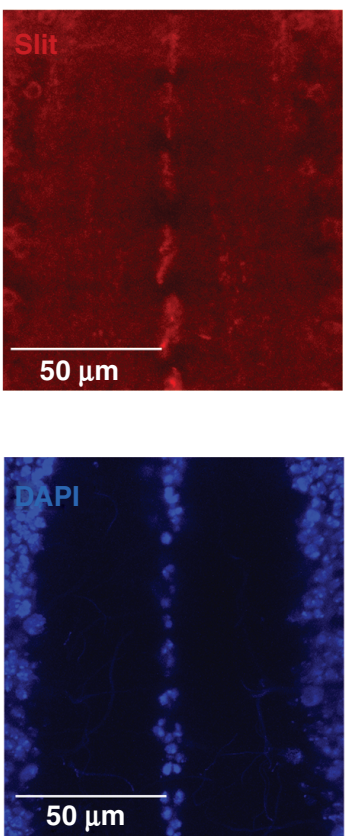

H3.3 WT

B
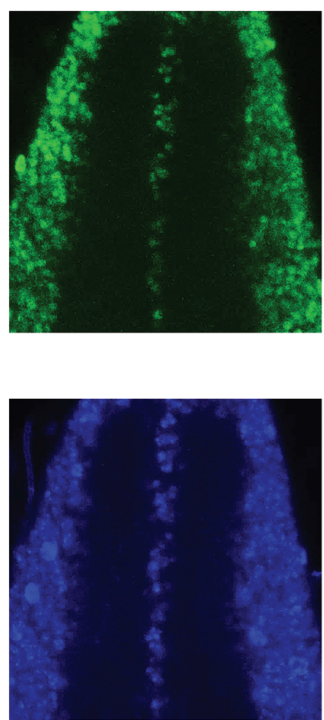

$\mathrm{F}$
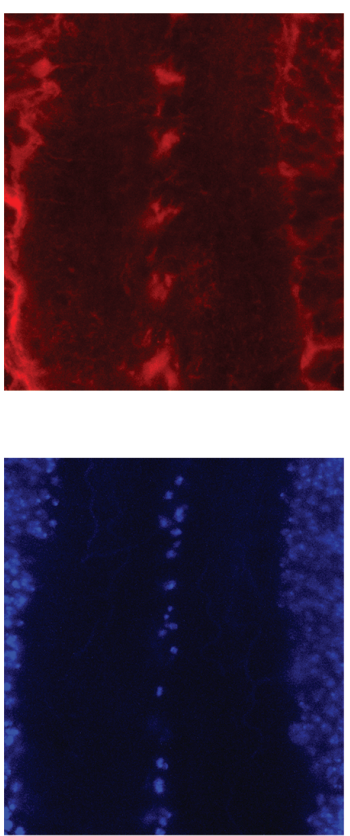

H3.3-K27M

C
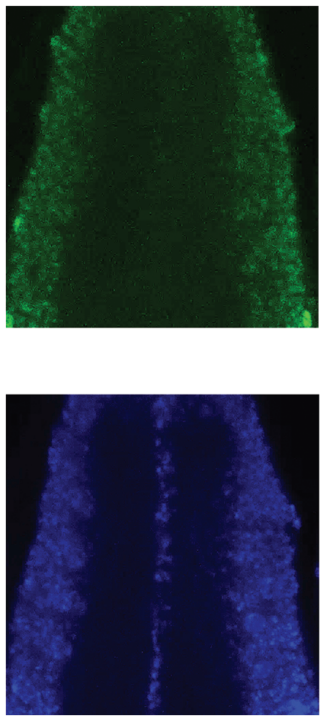

G

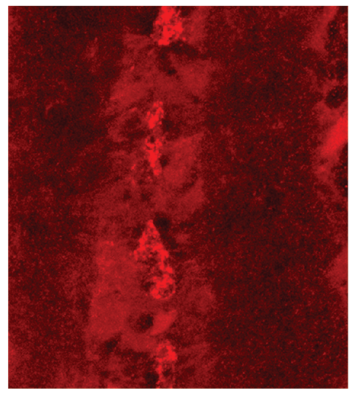

$\mathrm{H}$
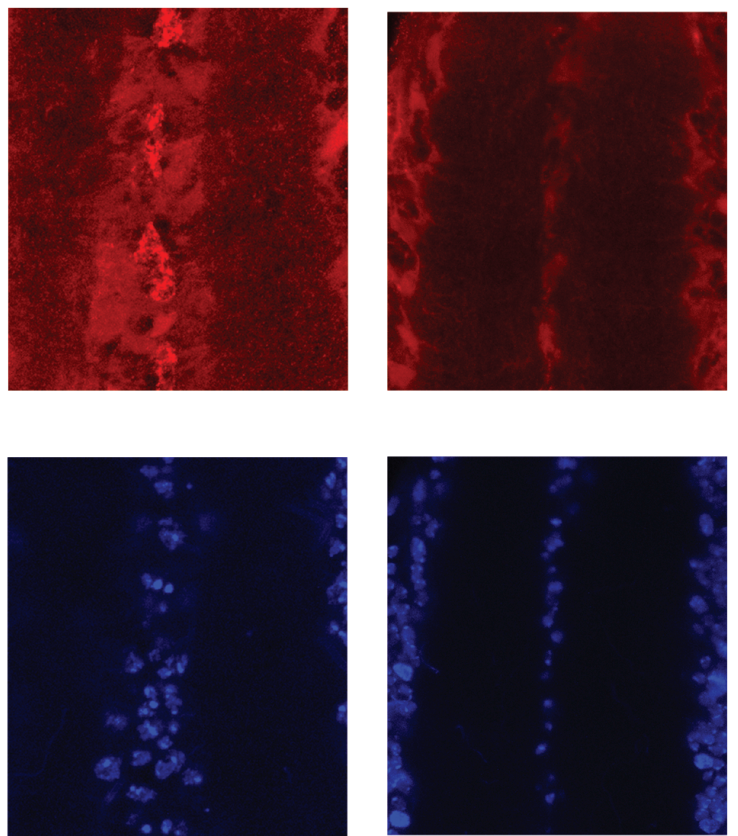

H3.3-G34R

D
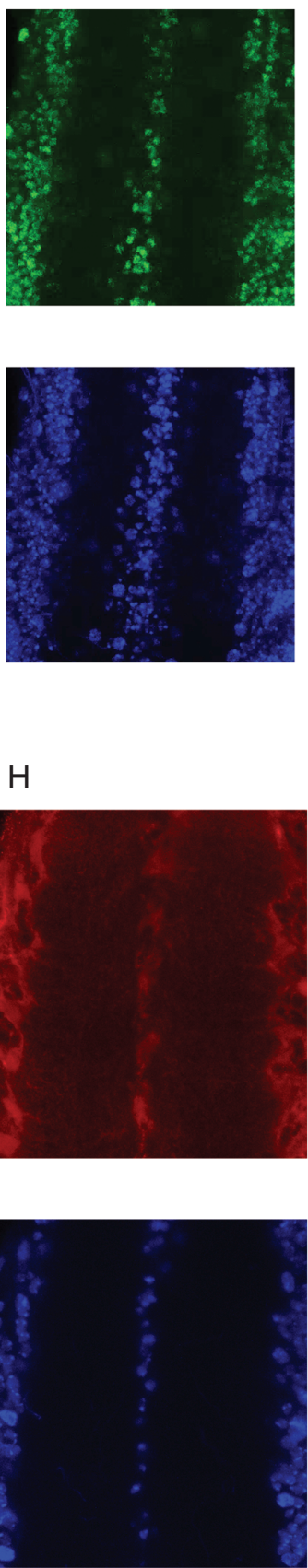

Fig. 2 Phenotype upon midline glia specific expression of wild-type and mutant H3.3 in Drosophila. H3K27me3 expression in third instar larval wild-type brains (control, A) compared with midline glia specific expression of H3.3 wild-type (WT, B), H3.3-K27M (C), and H3.3-G34R (D). Note loss of H3K27me3 in the midline only upon ubiquitous H3.3-K27M expression. Nuclear staining by 4',6'-diamidino-2-phenylindole (DAPI) serves as control. Expression of slit protein in third instar larval wild-type brains (control, E) compared with midline glia specific expression of H3.3 wild-type (WT, F), H3.3-K27M (G), and H3.3-G34R (H). Note perturbed distribution of slit protein only upon midline glia specific H3.3-K27M expression. Nuclear DAPI staining serves as control. For each condition, at least 5 animals were examined.

H3.3 wild-type modifier screen performed for comparison, shRNA knockdown of the majority of these genes did not cause a worse phenotype (see Supplementary Table 2).
Midline glia specific $\mathrm{H} 3.3-\mathrm{K} 27 \mathrm{M}$ expression in the screening strain (slit > UAS- H3.3-K27M/UAS-mCherry$R N A i)$ also produced pupal lethality under control 
A Ubiquitous H3.3-K27M modifier screen

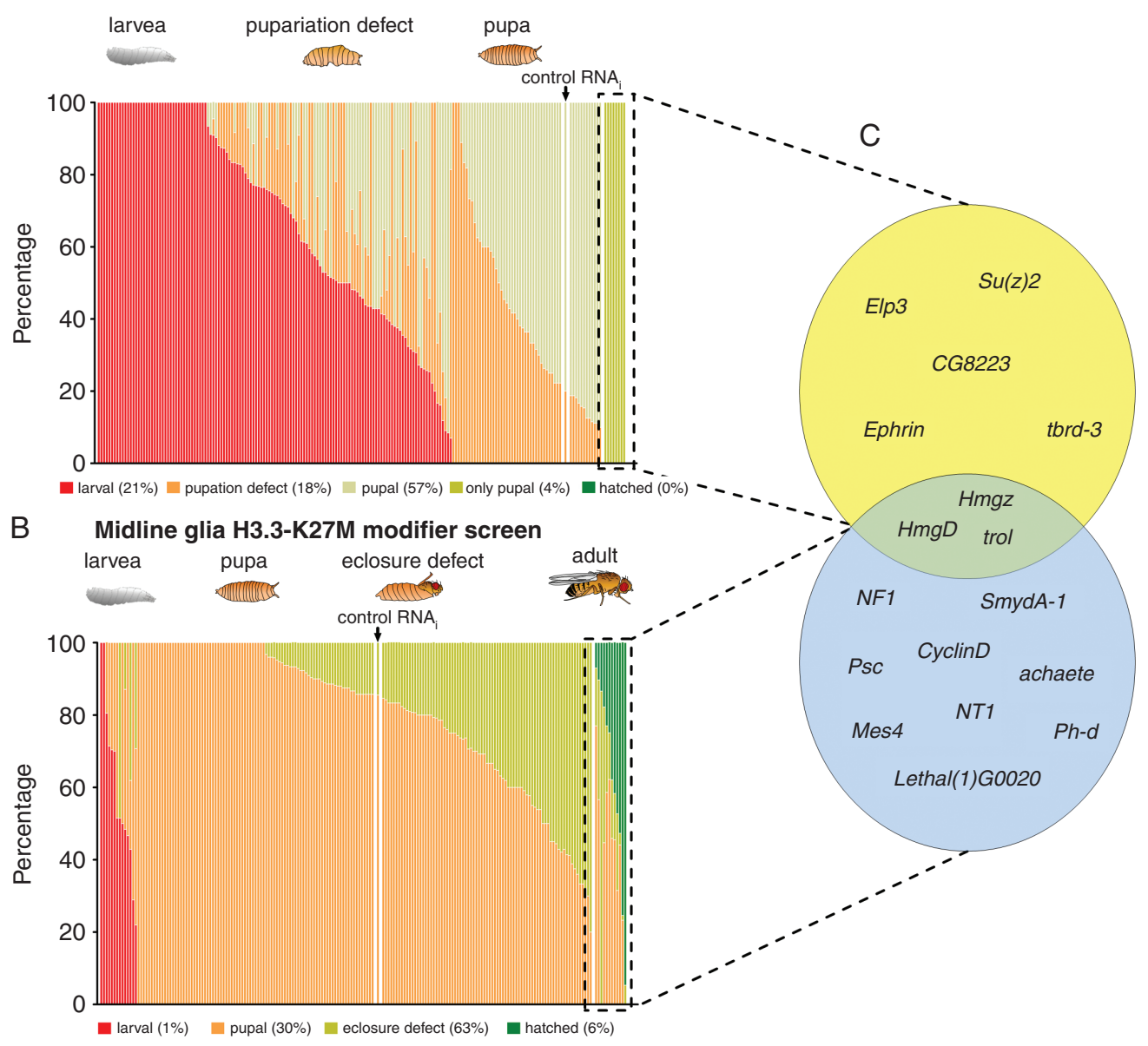

Fig. 3 Genes involved in the detrimental effects of H3.3-K27M expression. The potential of 194 candidate RNAi strains to shift the lethal phenotype encountered upon H3.3-K27M expression to earlier or later stages of development was investigated upon (A) ubiquitous and (B) midline glia specific H3.3-K27M expression. Each bar represents one of the 194 candidate genes, and the effect of shRNA knockdown on the lethal phenotype observed in the F1 generation is depicted. Note that shRNA knockdown of some genes caused a positive shift of the lethal phenotype compared with controls expressing H3.3-K27M and UAS-mCherry-RNAi (arrows), and the phenotype observed upon ubiquitous H3.3-K27M expression (larval lethality: red; pupal lethality with pupation defects: orange; pupal lethality: lime-green; only pupal lethality: olive green; hatched: green) was more severe than that observed upon midline glial H3.3-K27M expression (larval lethality: red; pupal lethality with pupation defects: orange; pupal lethality: lime-green; only pupal lethality: olive green; hatched: green). (C) Genes whose knockdown rescued pupation defects observed upon ubiquitous H3.3-K27M expression or lethality observed upon midline glial H3.3-K27M expression were examined in 3 independent experiments. Note that shRNA knockdown of $H m g Z, H m g D$, and trol shifted the phenotype to later stages of development upon both ubiquitous and midline glial specific H3.3-K27M expression.

conditions, with $86 \%(85-89 \%)$ of animals dying at the pupal stage and $14 \%(12-15 \%)$ of animals attempting but failing to eclose (Fig. 3B, control RNAi). Upon midline glia specific H3.3-K27M expression, shRNA knockdown of 12 of the 194 candidate genes shifted pupal lethality to later stages of development. Here, the rescue of the lethal phenotype was more pronounced, with as many as $95 \%$ of flies reaching adulthood (Fig. 3B, Table 2). These suppressor genes included 3 genes that had also shifted the phenotype upon ubiquitous H3.3-K27M expression: $\mathrm{HmgZ}$ (median hatching rate 95\% [93-97\%]), HmgD (10\% [6-15\%]), and trol (9\% [3-9\%]). Of note, knockdown of these genes not only rescued the phenotype upon ubiquitous H3.3-K27M expression, but also partly restored the loss of H3K27me3 (Supplementary Fig. 5), while location and quantity of Slit protein were not significantly altered (Supplementary Fig.s 6 and 7). Other genes whose knockdown suppressed the detrimental effects of midline glia specific H3.3-K27M expression were PRC1 components polyhomeotic distal ( $p h-d$; median hatching rate $64 \%$ [24-70\%]) and posterior sex combs (Psc; 42\% [17-83\%]), lysine acetyltransferase lethal (1) G0020 (29\% [26-59\%]), as well as downstream genes cyclin D (77\% [64-85\%]), SET and MYND domain containing, arthropod-specific, member 1 (SmydA-1; 30\% [28-68\%]), mesodermexpressed 4 (Mes4; 22\% [12-41\%]), neurotrophin 1 (NT1; $19 \%[15-35 \%])$, neurofibromin 1 (Nf1; 17\% [9-28\%]), and achaete (11\% [6-24\%]). In contrast, silencing of only 2 
Table 2 Genes shifting the lethal phenotype upon ubiquitous and midline glia specific expression of $\mathrm{H} 3.3-\mathrm{K}^{2} 7 \mathrm{M}^{*}$

\begin{tabular}{llll} 
Drosophila Gene & Human Orthologue & Phenotypic Shift & \\
HmgZ & HMGB3 & Ubiquitous & Midline glia specific \\
HmgD & HMGB2 & Ubiquitous & Midline glia specific \\
Su(z)2 & PCGF2 & Ubiquitous & \\
tbrd-3 & BRDT & Ubiquitous & \\
Elp3 & ELP3 & Ubiquitous & Midline glia specific \\
CG8223 & NASP & Ubiquitous & Midline glia specific \\
Ephrin & EFNB1 & Ubiquitous & Midline glia specific \\
trol & HSPG2 & Ubiquitous & Midline glia specific \\
ph-d & PHC2 & & Midline glia specific \\
Psc & $B M I 1$ & & Midline glia specific \\
Lethal (1) G0020 & $N A T 10$ & & Midline glia specific \\
Cyclin D & CCND1 & & Midline glia specific \\
SmydA-1 & $S M Y D 3$ & & Midline glia specific \\
Mes4 & POLE4 & & Midline glia specific \\
Neurotrophin 1 & $B D N F$ & & \\
NF1 & $N F 1$ & & \\
achaete & ASCL1 & & \\
\hline
\end{tabular}

${ }^{*}$ Drosophila genes whose knockdown shifted the lethal phenotype upon ubiquitous or midline glia specific expression of H3.3-K27M to later stages of development as well as their human orthologues.

candidate genes worsened the phenotype and produced complete larval lethality. These were jing and Su(var)3-9 (SupplementaryTable 2).

Potential effects of the 17 candidate genes involved in the detrimental effects of H3.3 K27M were also examined in an H3.3-G34R screening strain. Here, only 3 of the candidate genes (including $\mathrm{HmgZ}$ ) caused a shift of pupal lethality, suggesting that the majority of identified candidate genes rather play a role in the context of H3 K27M (SupplementaryTable 3).

Taken together, knockdown of 17 genes encoding histone modifying and chromatin remodeling proteins as well as downstream genes regulating cell differentiation and proliferation either rescued or shifted the lethal phenotype encountered upon ubiquitous and/or midline glia specific H3.3-K27M expression in our fly model to a later stage of development (Fig. 3C), suggesting that these genes play a functional role in the detrimental effects of mutant histone H3.3-K27M.

\section{Genes Involved in the Detrimental Effects of H3.3-K27M Expression Are Overexpressed in H3-K27M Mutant Diffuse Midline Gliomas}

To investigate if human orthologues of genes of functional relevance in the fly model might also play a role in the biology of H3-K27M mutant diffuse midline gliomas, RNA-seq data obtained from $5 \mathrm{H} 3-\mathrm{K} 27 \mathrm{M}$ mutant HGGs were compared with 4 HGGs without H3 mutations as well as with 3 nonneoplastic brain samples. Remarkably, human orthologues of identified candidate genes causing a positive shift in the fly model displayed, as a set, elevated gene expression levels in $\mathrm{H} 3-\mathrm{K} 27 \mathrm{M}$ mutant HGGs compared with $\mathrm{H} 3$ wild-type HGG $(P<0.01)$ as well as with normal brain samples ( $P<0.001$; Fig. 4A). Singlesample GSEA further supported a difference between gene expression levels in $\mathrm{H} 3-\mathrm{K} 27 \mathrm{M}$ mutant HGGs compared with normal brain samples $(P<0.05$; Fig. $4 \mathrm{~B})$, while differences between H3-K27M mutant HGGs and H3 wild-type HGG were less pronounced. Human orthologues of 5 genes (ie, ASCL1, HMGB2, CCND1, EFNB1, and HSPG2) were significantly upregulated in H3-K27M HGG compared with normal brain samples (Fig. 4C). Furthermore, 14 of 17 human orthologues of candidate genes also displayed higher expression levels (fold change up to 2.53) in H3-K27M HGG compared with H3 wild-type HGG (Fig. 4D), but these differences did not reach significance. Orthologues of the remaining genes from the modifier screen were not significantly overexpressed as a set (96 upregulated and 63 downregulated, $P=0.08$ ). On protein level, overexpression of CCND1, BMI1, and HMGB2 was confirmed in an unrelated series of H3 K27M mutated HGGs $(N=8)$, CCND1 being significantly overexpressed compared with histone $\mathrm{H} 3$ wild-type HGGs (8/8 vs $3 / 9$ tumors; chisquare $=8.242, P<0.01$; Fig. 5 ).

Taken together, these findings further corroborate the relevance of genes identified in the fly model in the biology of H3-K27M mutant HGGs.

\section{Discussion}

We generated a tissue-specific transgenic fly model of H3.3-K27M mutagenesis. In this model, ubiquitous and midline glia expression of H3.3-K27M caused pupal lethality, induced morphological alterations, and, as expected, decreased overall levels of H3K27me3 in tissues expressing H3.3-K27M. The lethal phenotype we observed 
A

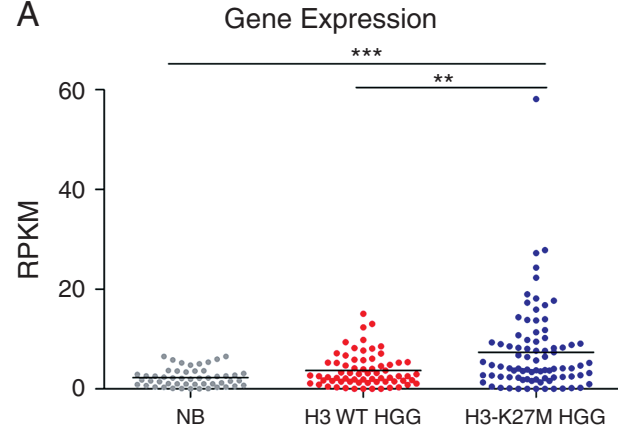

C

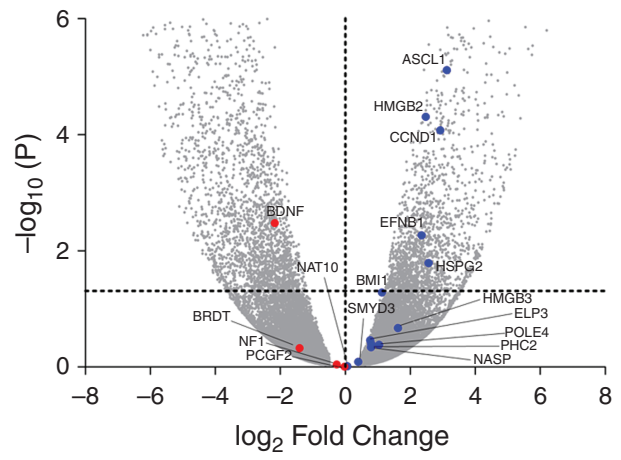

B

Enrichment

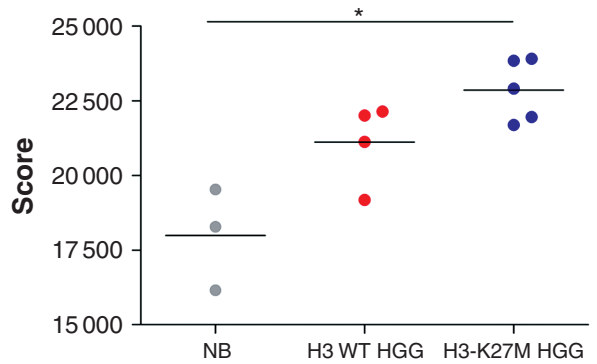

D

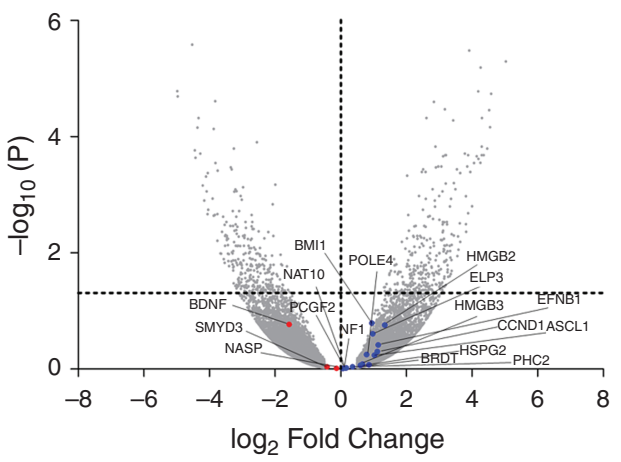

Fig. 4 Orthologues of Drosophila candidate genes are overexpressed in H3-K27M-HGG. (A) Expression of human orthologues of all fly candidate genes shifting the lethal phenotype to later stages of development are overrepresented in H3-K27M HGG ( $N=5$ ) compared with $\mathrm{H} 3$ wild-type HGG $(N=4)$ and normal brain samples $\left(N=3 ;{ }^{* *} P<0.01 ;{ }^{* * *} P<0.001\right)$. The number of detected reads (RPKM) for each transcript is shown for each tumor sample. (B) Single-sample GSEA of human orthologues of the genes identified in the fly model, as in $(A)\left({ }^{*} P<0.05\right)$. (C) Volcano plot of differentially expressed genes in H3-K27M HGG compared with normal brain samples. Highlighted are human orthologues of Drosophila genes inducing a positive shift upon downregulation in H3.3-K27M expressing flies (upregulated: blue; downregulated: red). Note that expression of 5 individual genes (ASCL1, HMGB2, CCND1, EFNB1, HSPG2) is significantly upregulated. (D) Volcano plot of differentially expressed genes in H3-K27M HGG compared with $\mathrm{H} 3$ wild-type HGG. Highlighted are human orthologues of Drosophila genes inducing a positive shift upon downregulation in $\mathrm{H} 3.3-$ K27M expressing flies (upregulated: blue; downregulated: red).

was shifted by genes encoding histone interacting and chromatin remodeling proteins, but also by genes regulating cell differentiation and proliferation, indicative of their potential role in mediating the detrimental effects of H3.3$\mathrm{K} 27 \mathrm{M}$ in vivo. Notably, several of these modifier genes identified in the fruit fly screen were also overexpressed in human H3.3-K27M tumors, supporting their relevance to human disease. Remarkably, expression of H3.3-K27M, but not H3.3-G34R or wild-type H3.3, in midline glia was deleterious consistent with a specific effect of this mutation in that brain site and in keeping with human data showing the specific localization of H3.3-K27M mutant tumors in the brain midline in human gliomas. Altogether, these results make a compelling case for the relevance of this model to human H3K27M mutant gliomas.

Midline glia specific expression of H3.3-K27M perturbed the localization of Slit, which plays a key role in axonal guidance. In the larval fly brain, midline glia cells are the main source of Slit. ${ }^{27}$ In humans, a role of Slit2 has been also suggested in glioma cell migration, ${ }^{26}$ and dysregulated Slit expression has been implicated in the biology of a variety of cancers, ${ }^{28}$ including in H3-K27M mutant
HGGs. ${ }^{29}$ Our finding that midline glia specific expression of H3.3-K27M was associated with both decreased H3K27me3 as well as increased Slit protein expression is potentially in line with a dominant inhibition of PRC2 and suggests a role of polycomb repression in midline glia cell function. Indeed, in cells, PRC2 binds to the Slit2 promoter and promotes trimethylation of $\mathrm{H} 3 \mathrm{~K} 27$ and gene silencing, which is lost following K27M-H3.3 expression. ${ }^{30}$ On the other hand, knockdown of slit in the fly model did not shift the lethal phenotype, arguing against a direct role of slit in the lethal phenotype observed upon midline glial specific H3-K27M overexpression. This is well in line with our finding that human slit orthologues SLIT1, SLIT2, and SLIT3 were not differentially expressed in H3-K27M mutant gliomas.

Ubiquitous expression of H3.3-K27M caused the formation of melanotic masses, which is a well-accepted cancer equivalent in Drosophila and involves hemocyte-mediated immune responses. ${ }^{31}$ Lack of histone variant $\mathrm{H} 2 \mathrm{AV}$ has also been shown to cause formation of melanotic masses, ${ }^{32}$ and it has been suggested that polycomb repression might require 2 independent pathways, one involving $\mathrm{H} 2 \mathrm{AV}$ recruitment and a second one leading to H3K27me3. ${ }^{33}$ Our 


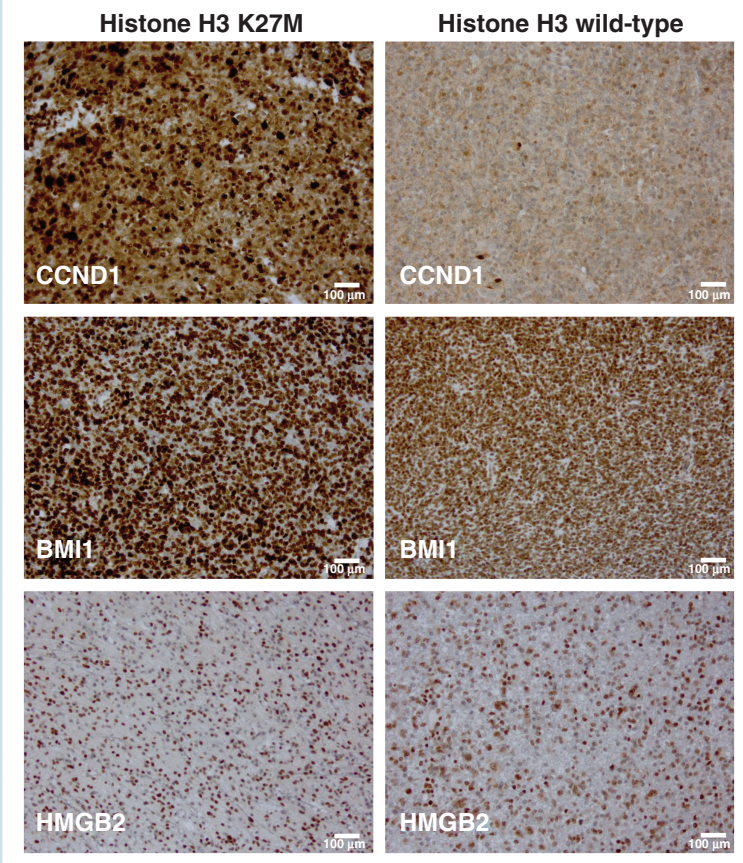

Fig. 5 Protein expression of CCND1, BMI1, and HMGB2 in histone H3-K27M HGGs compared with histone H3 wild-type HGGs. In H3-K27M mutated HGGs ( $N=8$ ), overexpression of CCND1, BMI1, and HMGB2 was confirmed on protein level, CCND1 also being significantly overexpressed compared with histone $\mathrm{H} 3$ wild-type $\mathrm{HGGs}$ ( $8 / 8$ vs $3 / 9$ tumors; chi-square $=8.242, P<0.01)$. Representative staining results are shown.

finding that transgenic expression H3.3-K27M was associated with both H3K27me3 loss and melanization further supports this view and strongly suggests a role of polycomb de-repression in melanization.

We observed that knockdown of several PRC1/PRC2 genes shifted the lethal phenotype encountered upon expression of H3.3-K27M. Silencing of PRC2 members $E(z)$, Caf1-55, $S u(z) 12$, and jing shifted the lethal phenotype to earlier stages of development, which is well in line with a synergistic effect with H3.3-K27M, known to cause disturbed PRC2 repression. ${ }^{15}$ Interestingly, $P c$ represented the only PRC1 component shifting the lethal phenotype to earlier stages of development, which might well be related to reduced $\mathrm{H} 3 \mathrm{~K} 27 \mathrm{me} 3$ and reduced $\mathrm{PRC} 1$ recruitment under these conditions. ${ }^{34-36}$ In contrast, downregulation of other PRC1 members, namely Psc, Su(z)2 and ph-d, produced the opposite effect of PRC2 knockdown, shifting the lethal phenotype to later stages of development. The hierarchical model of polycomb repression postulates PRC2 activity to be crucial for PRC1 recruitment by direct interaction of the PC subunit and H3K27me3. ${ }^{34-36}$ However, other studies also suggested independent binding sites as well as bidirectional recruitment mechanisms for PRC1 and PRC2. ${ }^{37,38}$ Furthermore, a genetic study in Drosophila revealed 2 distinct classes of PRC1 target genes, one (class I) requiring all PRC1 components and a second class (class II) being more dependent on the presence of $p h-d, P s c$, and $S u(z) 2$ rather than $P C$ and other PRC1 components. ${ }^{39}$ Our findings are more consistent with PRC1 interacting differently with distinct classes of target genes, and demonstrate for the first time a functional involvement for PRC1 in the detrimental effects of H3.3-K27M expression. Notably, they mirror recent findings of an effect of $\mathrm{PRC} 1$ in mediating the oncogenesis of another $\mathrm{H} 3$ variant mutation, $\mathrm{H} 3 \mathrm{~K} 36 \mathrm{M} .{ }^{40}$

Other genes involved in the effects of H3.3-K27M in our fly model were $H m g Z$ and $H m g D$, members of the high mobility group box protein family which are known to participate in chromatin remodeling by interacting with trithorax group protein Brahma. ${ }^{41}$ Besides polycomb repression, the trithorax group of transcriptional activators is thought to organize global gene expression in collaboration with polycomb group proteins. Our results thus suggest a possible attenuation of altered polycomb repression in K27M mutants by Brahma-dependent trithorax group activation aided by high mobility group box proteins.

Knockdown of 4 genes associated with posttranslational modifications of histones also shifted lethality to later stages of development. Those included a lysine methyltransferase $(S m y d A-1)^{42}$ and 3 genes implicated in histone acetylation (Elp3, lethal (1) G0020, and CG8223), suggesting a role of histone acetyl- and methyltransferases in the detrimental effects of H3.3-K27M expression. Other modifier genes were not directly linked to chromatin remodeling or histone interaction, but rather involved more downstream pathways. These candidates consisted of 2 cell cycle regulators, $C y c D$ and trol, ${ }^{43,44}$ as well as NT1, achaete, and Ephrin, 3 genes playing important roles in nervous system development. ${ }^{45-47}$ Additionally, Mes4, which is known to be involved in early dorsal-ventral patterning, ${ }^{48}$ the germline specific gene $t b r d-3,49$ and Nf1 shifted the lethal phenotype to later stages of development. The finding that knockdown of $N f 1$, which is a well-established tumor suppressor, counteracts the detrimental effects of H3.3-K27M is unexpected. However, similar results have been observed in a fly model of atypical teratoid/rhabdoid tumor and have been linked to Hippo pathway activation. ${ }^{22}$

The majority of candidate target genes shifted the phenotype only upon ubiquitous or midline glial cellspecific expression. This is a common finding, which could be related to different drivers or GAL4 dosage effects. Furthermore, midline glial specific knockdown of some genes might be easier to rescue than upon ubiquitous knockdown. The number of human tumor samples available for the present study was relatively small, and relatively few candidate genes were found to be differentially expressed when comparing gene expression data from H3-K27M mutated and wild-type HGGs with normal brain samples. Even though all 3 normal brain samples were pediatric (median age of the patients: $7.4 \mathrm{y}$ ), this could be also related to the fact that only 2 of the 3 tissue samples were of midline location. Normal age-matched tissues of the midline are hard to obtain, but examination of larger cohorts including such matched controls would be highly desirable. Apart from these considerations, our gene expression data compare well with a dataset previously published by Funato et al. ${ }^{16}$ Although the complete differential expression results were not included in that study, raw expression profiling data were made 
publicly available by the authors. We retrieved the data, reanalyzed them, and found significant upregulation ( $P$ $<0.05$ ) of 4 human orthologues of the candidate genes in $\mathrm{K} 27 \mathrm{M}$ mutant compared with mock neuronal precursor cells: BMI1, CCND1, PCGF2, and SMYD3. Notably, CCND1 was also found to be one of the most significantly upregulated genes in the H3-K27M HGG versus normal brain comparison described in our paper. In line with these findings, differential expression of CCND1 in H3-K27M HGG could also be confirmed on protein level.

Patients harboring diffuse midline glioma H3-K27M mutant (WHO grade IV) carry a dismal prognosis. ${ }^{50}$ Early-stage clinical trials of a histone deacetylase inhibitor, panobinostat (NCT02717455), and an H3.3-K27M peptide vaccine (NCT02960230) as therapeutic agents are under way. It is noteworthy that among the genes involved in the detrimental effects of H3.3-K27M in the fly model are some others whose products are potentially targetable by small molecules. These warrant further investigations as they may provide needed alternative therapeutic strategies in a deadly disease. The majority of H3-K27M mutated HGGs carry mutations of the H3F3A gene encoding for histone H3.3, but biological and clinical differences between H3.3-K27M and H3.1-K27M mutated HGGs have been suggested. It will therefore be of interest to also develop an H3.1-K27M fly model in order to further study similarities and differences of H3.3-K27M and H3.1-K27M. Furthermore, the fly model could be also used to study the functional role of other genes and pathways known to be relevant in the biology of HGGs.

In conclusion, we uncover novel genes functionally involved in H3K27M mutagenesis using a transgenic Drosophila model. Our results suggest that these fly models are powerful tools for the identification of functionally relevant genes involved in the detrimental effects of mutant $\mathrm{H} 3$ and may aid to discover relevant genes and pathways in deadly human cancers.

\section{Supplementary Material}

Supplementary data are available at Neuro-Oncology online.

\section{Keywords}

chromatin remodeling | diffuse midline glioma | histone H3-K27M | PRC2

\section{Funding}

M.H. is supported by IZKF Münster ( $\mathrm{Ha} 3 / 019 / 15)$ and Deutsche Forschungsgemeinschaft (DFG HA 3060/10-1). P.L. is supported by Canadian Institutes for Health Research (CIHR) grant IOP107345. This project received infrastructural support from the Medical Faculty of the University Münster (Technology Platform "Drosophila"). M.S is supported by the American University of Beirut Medical Practice Plan (MPP). C.K. is supported by Natural Sciences and Engineering Research Council (NSERC ) RGPIN-2016-04911. Additional funding was from the US National Institutes of Health (NIH) grant P01CA196539 (to N.J); the Canadian Institutes for Health Research (CIHR) grant MOP 286756 (to N.J.); I-CHANGE consortium; the Fonds de Recherche du Québec-Santé (salary awards, N.J., C.L.K., N.D.J.). Computational infrastructure was provided by Compute Canada. All sequencing data have been deposited on GEO (accession number GSE95169).

Conflict of interest statement. The authors have no competing financial interests to declare.

Authorship statement. $\mathrm{NJ}, \mathrm{PL}$, and $\mathrm{MH}$ jointly conceived the study. JB, AC, IT, KT, ND, MS, CK, AJ, PL, NJ, and MH were involved in the acquisition, analysis, or interpretation of data for the work. JB drafted the manuscript and all other co-authors revised it critically for important intellectual content. All authors have read and approved the final version.

\section{References}

1. Wu G, Broniscer A, McEachron TA, et al. Somatic histone H3 alterations in pediatric diffuse intrinsic pontine gliomas and non-brainstem glioblastomas. Nat Genet. 2012;44(3):251-253.

2. Schwartzentruber J, Korshunov A, Liu XY, et al. Driver mutations in histone $\mathrm{H} 3.3$ and chromatin remodelling genes in paediatric glioblastoma. Nature. 2012;482(7384):226-231.

3. Khuong-Quang DA, Buczkowicz P, Rakopoulos P, et al. K27M mutation in histone H3.3 defines clinically and biologically distinct subgroups of pediatric diffuse intrinsic pontine gliomas. Acta Neuropathol. 2012;124(3):439-447.

4. Wu G, Diaz AK, Paugh BS, et al. The genomic landscape of diffuse intrinsic pontine glioma and pediatric non-brainstem high-grade glioma. Nat Genet. 2014;46(5):444-450.

5. Taylor KR, Mackay A, Truffaux N, et al. Recurrent activating ACVR1 mutations in diffuse intrinsic pontine glioma. Nat Genet. 2014;46(5):457-461.

6. Fontebasso AM, Papillon-Cavanagh S, Schwartzentruber J, et al. Recurrent somatic mutations in ACVR1 in pediatric midline high-grade astrocytoma. Nat Genet. 2014;46(5):462-466.

7. Buczkowicz P, Hoeman C, Rakopoulos P, et al. Genomic analysis of diffuse intrinsic pontine gliomas identifies three molecular subgroups and recurrent activating ACVR1 mutations. Nat Genet. 2014;46(5):451-456.

8. Liu XY, Gerges N, Korshunov A, et al. Frequent ATRX mutations and loss of expression in adult diffuse astrocytic tumors carrying IDH1/IDH2 and TP53 mutations. Acta Neuropathol. 2012;124(5):615-625.

9. Louis DN, Perry A, Reifenberger G, et al. The 2016 World Health Organization classification of tumors of the central nervous system: a summary. Acta Neuropathol. 2016;131(6):803-820. 
10. Fontebasso AM, Jabado N. Pediatric brain tumors: genomics and epigenomics pave the way. Crit Rev Oncog. 2015;20(3-4):271-299.

11. Bechet D, Gielen GG, Korshunov A, et al. Specific detection of methionine 27 mutation in histone 3 variants (H3K27M) in fixed tissue from high-grade astrocytomas. Acta Neuropathol. 2014;128(5):733-741.

12. Fontebasso AM, Schwartzentruber J, Khuong-Quang DA, et al. Mutations in SETD2 and genes affecting histone H3K36 methylation target hemispheric high-grade gliomas. Acta Neuropathol. 2013;125(5):659-669.

13. Szenker E, Ray-Gallet D, Almouzni $G$. The double face of the histone variant H3.3. Cell Res. 2011;21(3):421-434.

14. Tessarz P, Kouzarides T. Histone core modifications regulating nucleosome structure and dynamics. Nat Rev Mol Cell Biol. 2014;15(11):703-708.

15. Lewis PW, Müller MM, Koletsky MS, et al. Inhibition of PRC2 activity by a gain-of-function $\mathrm{H} 3$ mutation found in pediatric glioblastoma. Science. 2013;340(6134):857-861.

16. Mohammad F, Weissmann S, Leblanc B, et al. EZH2 is a potential therapeutic target for H3K27M-mutant pediatric gliomas. Nat Med. 2017;23(4):483-492.

17. Funato $K$, Major $T$, Lewis PW, Allis CD, Tabar V. Use of human embryonic stem cells to model pediatric gliomas with $\mathrm{H} 3.3 \mathrm{~K} 27 \mathrm{M}$ histone mutation. Science. 2014;346(6216):1529-1533.

18. Sturm D, Witt $H$, Hovestadt $V$, et al. Hotspot mutations in H3F3A and IDH1 define distinct epigenetic and biological subgroups of glioblastoma. Cancer Cell. 2012;22(4):425-437.

19. Piunti A, Hashizume R, Morgan MA, et al. Therapeutic targeting of polycomb and BET bromodomain proteins in diffuse intrinsic pontine gliomas. Nat Med. 2017;23(4):493-500.

20. Nikbakht H, Panditharatna E, Mikael LG, et al. Spatial and temporal homogeneity of driver mutations in diffuse intrinsic pontine glioma. Nat Commun. 2016;7:11185.

21. Pathania M, De Jay N, Maestro N, et al. H3.3K27M cooperates with Trp53 Loss and PDGFRA gain in mouse embryonic neural progenitor cells to induce invasive high-grade gliomas. Cancer Cell. 2017;32(5):684-700.e9.

22. Jeibmann A, Eikmeier $K$, Linge $A$, et al. Identification of genes involved in the biology of atypical teratoid/rhabdoid tumours using Drosophila melanogaster. Nat Commun. 2014;5:4005.

23. Herz HM, Morgan M, Gao X, et al. Histone H3 lysine-to-methionine mutants as a paradigm to study chromatin signaling. Science. 2014;345(6200):1065-1070.

24. Bolger AM, Lohse M, Usadel B. Trimmomatic: a flexible trimmer for Illumina sequence data. Bioinformatics. 2014;30(15):2114-2120.

25. Barbie DA, Tamayo P, Boehm JS, et al. Systematic RNA interference reveals that oncogenic KRAS-driven cancers require TBK1. Nature. 2009;462(7269):108-112.

26. Mertsch S, Schmitz N, Jeibmann A, Geng JG, Paulus W, Senner V. Slit2 involvement in glioma cell migration is mediated by Robo1 receptor. $J$ Neurooncol. 2008;87(1):1-7.

27. Kidd T, Bland KS, Goodman CS. Slit is the midline repellent for the robo receptor in Drosophila. Cell. 1999;96(6):785-794.

28. Gara RK, Kumari S, Ganju A, Yallapu MM, Jaggi M, Chauhan SC. Slit/ Robo pathway: a promising therapeutic target for cancer. Drug Discov Today. 2015;20(1):156-164.

29. Paugh BS, Qu C, Jones $C$, et al. Integrated molecular genetic profiling of pediatric high-grade gliomas reveals key differences with the adult disease. J Clin Oncol. 2010;28(18):3061-3068.

30. Yu J, Cao Q, Yu J, et al. The neuronal repellent SLIT2 is a target for repression by EZH2 in prostate cancer. Oncogene. 2010;29(39):5370-5380.
31. Minakhina S, Steward R. Melanotic mutants in Drosophila: pathways and phenotypes. Genetics. 2006;174(1):253-263.

32. Grigorian M, DeBruhl H, Lipsick JS. The role of variant histone H2AV in Drosophila melanogaster larval hematopoiesis. Development. 2017;144(8):1441-1449.

33. Swaminathan J, Baxter EM, Corces VG. The role of histone H2Av variant replacement and histone $\mathrm{H} 4$ acetylation in the establishment of Drosophila heterochromatin. Genes Dev. 2005;19(1):65-76.

34. Cao R, Wang L, Wang $H$, et al. Role of histone H3 lysine 27 methylation in Polycomb-group silencing. Science. 2002;298(5595): 1039-1043.

35. Wang $\mathrm{H}$, Wang $\mathrm{L}$, Erdjument-Bromage $\mathrm{H}$, et al. Role of histone H2A ubiquitination in Polycomb silencing. Nature. 2004;431(7010): 873-878.

36. Boyer LA, Plath K, Zeitlinger J, et al. Polycomb complexes repress developmental regulators in murine embryonic stem cells. Nature. 2006;441(7091):349-353.

37. Ku M, Koche RP, Rheinbay E, et al. Genomewide analysis of PRC1 and PRC2 occupancy identifies two classes of bivalent domains. PLoS Genet. 2008;4(10):e1000242.

38. Blackledge NP, Farcas AM, Kondo T, et al. Variant PRC1 complex-dependent H2A ubiquitylation drives PRC2 recruitment and polycomb domain formation. Cell. 2014;157(6):1445-1459.

39. Gutiérrez L, Oktaba K, Scheuermann JC, Gambetta MC, Ly-Hartig N, Müller J. The role of the histone H2A ubiquitinase Sce in Polycomb repression. Development. 2012;139(1):117-127.

40. Lu C, Jain SU, Hoelper D, et al. Histone H3K36 mutations promote sarcomagenesis through altered histone methylation landscape. Science. 2016;352(6287):844-849.

41. Ragab A, Thompson EC, Travers AA. High mobility group proteins HMGD and HMGZ interact genetically with the Brahma chromatin remodeling complex in Drosophila. Genetics. 2006;172(2):1069-1078.

42. Jiang $F$, Liu 0 , Wang $Y$, et al. Comparative genomic analysis of SET domain family reveals the origin, expansion, and putative function of the arthropod-specific SmydA genes as histone modifiers in insects. Gigascience. 2017;6(6):1-16.

43. Datar SA, Jacobs HW, de la Cruz AF, Lehner CF, Edgar BA. The Drosophila cyclin D-Cdk4 complex promotes cellular growth. EMBO J. 2000;19(17):4543-4554.

44. Park $Y, \mathrm{Ng} \mathrm{C}$, Datta $\mathrm{S}$. Induction of string rescues the neuroblast proliferation defect in trol mutant animals. Genesis. 2003;36(4):187-195.

45. Zhu B, Pennack JA, McQuilton P, et al. Drosophila neurotrophins reveal a common mechanism for nervous system formation. PLoS Biol. 2008:6(11):e284

46. García-Bellido A, de Celis JF. The complex tale of the achaete-scute complex: a paradigmatic case in the analysis of gene organization and function during development. Genetics. 2009;182(3):631-639.

47. Cramer KS, Miko IJ. Eph-ephrin signaling in nervous system development. F1000Res. 2016:5:1-8.

48. Stathopoulos A, Van Drenth M, Erives A, Markstein M, Levine M. Whole-genome analysis of dorsal-ventral patterning in the Drosophila embryo. Cell. 2002;111(5):687-701.

49. Theofel I, Bartkuhn M, Hundertmark T, et al. tBRD-1 selectively controls gene activity in the Drosophila testis and interacts with two new members of the bromodomain and extra-terminal (BET) family. PLoS One. 2014;9(9):e108267.

50. Karremann M, Gielen GH, Hoffmann M, et al. Diffuse high-grade gliomas with $\mathrm{H} 3 \mathrm{~K} 27 \mathrm{M}$ mutations carry a dismal prognosis independent of tumor location. Neuro Oncol. 2018;20(1):123-131. 